\title{
EXTRACTING KINEMATICS FROM SPECTRA: A NEW METHOD AND NGC 4406
}

\author{
T. B. WILLIAMS \\ Dept. of Physics and Ástronomy, Rutgers University, \\ Piscataway, NJ 08855-0849, USA. \\ P. SAHA \\ CITA, University of Toronto, \\ 60 St. George Street, Toronto, Ontario M5S 1A7, Canada.
}

\section{Introduction}

The observed spectrum of a galaxy is the composite spectrum of its stellar population broadened by the stellar velocities. Our topic is the extraction of the broadening functions (i.e., line-of-sight velocity distributions) from spectra. In the past, observers tried to extract only mean velocities and dispersions. But recent work on deconvolution [Franx \& Illingworth ApJL 327, L55 (1989), Bender A\&A 229, 441 (1990), Rix \& White MNRAS 254, 389 (1992), van der Marel \& Franx $A p J$, to appear] shows that more information can be recovered. The general idea is to compare a galaxy spectrum with one or more stellar 'template' spectra; but various methods differ widely in the treatment of noise and the control of sources of systematic error.

We have developed a new deconvolution method, though drawing on previous work. Here we show rotation curves, dispersions, and deviations from Gaussian broadening functions for the major axis of the E3 NGC 4406.

\section{Observations}

The galaxies were observed using the Large Cassegrain Spectrograph and on the McDonald Observatory 2.7 meter telescope on February $8-9,1992$. The spectra cover the wavelength region $4800 \AA$ to $5500 \AA$ with a resolution of $1.8 \AA$; the seeing ranged from 1.5 to 2.5 arcseconds. The galaxies NGC $1439,1700,3379,4261$, and 4406 were observed at $2-5$ different position angles. Some preliminary results for NGC 4406 are presented here.

\section{Analysis method}

We have used the form of van der Marel and Franx for the broadening function, with four parameters $V, \sigma, h_{3}, h_{4}-h_{3}$ measures the skewness and $h_{4}$ the kurtosis (relative to a Gaussian). In other respects, our method is closest to Rix and White's-it does the fitting in pixel space rather than Fourier space (so pixels with higher $S / N$ automatically get more weight), and includes a simple population synthesis. However, we introduce two improvements:

1. Our 'pixels' for the fit really are the pixels on which the galaxy spectrum is recorded, not a rebinned version. This avoids correlations in the noise introduced by rebinning.

2. We assume that the noise in the data is Gaussian, but we don't assume that the errors in the parameter fits are Gaussian. Previous approaches supply parameter values and formal Gaussian error estimates. We use a Bayesian 
method to compute a probability distribution for the parameters. The estimates shown in here are the medians and $66 \%$ boundaries of such probability distributions.

\section{Conclusions}

Significantly non-Gaussian line-of-sight velocity distributions are the rule rather than the exception. The deviations from Gaussian provide new input for modelling the dynamics of galaxies. Ignoring these deviations is likely to produce systematic errors in estimates of dispersions.

The deconvolution method we have developed has the advantages that (i) the 'fitting' process is very transparent; (ii) prior information, if available, can be incorporated through the Bayesian prior probability; and (iii) the error analysis is more general (and, we believe, more reliable) than for previous methods.

Possible applications: (i) The search for central black-holes. (ii) It is now feasible to map potentials from discrete velocity measurements (see Merritt, preprint 1992); broadening functions (such as presented here) could probably also be used for this purpose.

Programs are available to anyone interested.

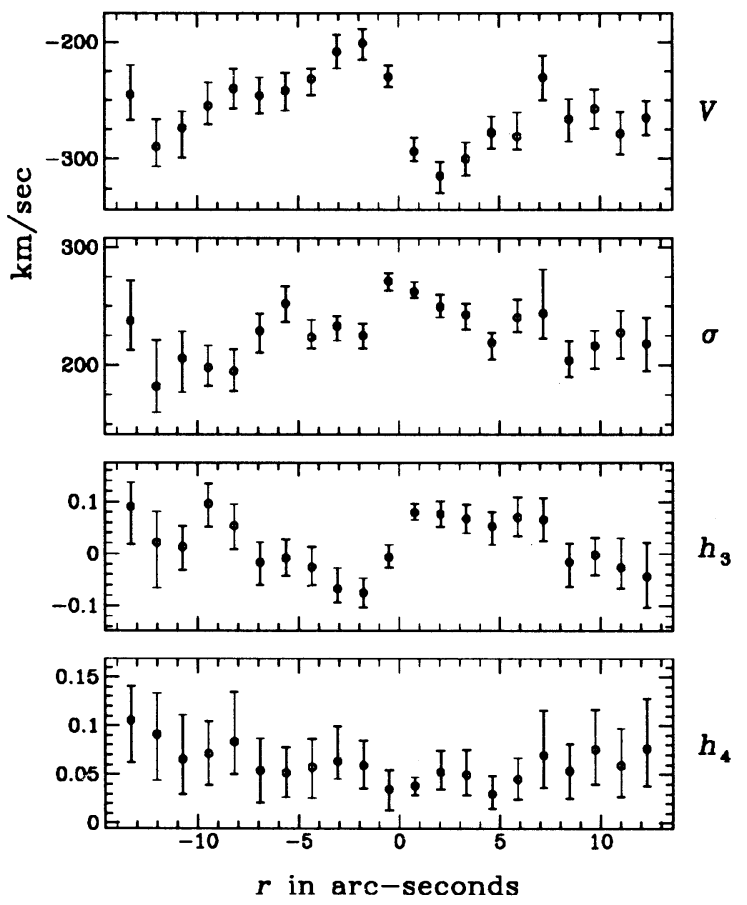

Figure 1. NGC 4406 (M86) along the major axis. The plot of $V$ shows a counterrotating core. Note also the antisymmetry of the skewness $\left(h_{3}\right)$ plot as we move away from the centre. The latter seems to be a fairly common feature in ellipticals; it may result from a combination of velocity anisotropy and rotation or it may imply two kinematically distinct components-it is not yet clear which. 